\title{
Evaluación del impacto económico de producción de pastos mejorados perennes en los productores agropecuarios
}

Evaluation of the economic impact of production of improved perennial pastures in agricultural producers

Avaliação do impacto econômico da produção de melhoria de pastagens perenas em productores agrícolas

\author{
Víctor Raúl Cotrina- Cabello \\ victor.cotrina3@gmail.com \\ https://orcid.org/0000-0002-0518-7308
}

Universidad Nacional Hermilio Valdizan, Huánuco, Perú

\author{
Santiago Oscar Puente-Segura \\ santiagio.puente@unh.edu.pe \\ https://orcid.org/0000-0002-0518-2407
}

Universidad Nacional de Huancavelica, Huancavelica, Perú

\section{Guillermo Gomer Cotrina Cabello \\ guillermo.cotrina@unh.edu.pe \\ https://orcid.org/0000-0002-3226-2094}

Universidad Nacional de Huancavelica, Huancavelica, Perú

Artículo recibido 17 de marzo 2021 / Arbitrado y aceptado 07 de abril 2021 / Publicado 04 de mayo 2021

\section{RESUMEN}

El objetivo de investigación fue evaluar el impacto económico de la producción de pastos mejorados en los productores agropecuarios, los métodos usado fueron deductivo inductivo con su técnica de revisión documental y sus respectivos instrumentos de la estadística descriptiva, los resultados de los aspectos sociales; en la variable nivel de instrucción de los productores tiene poca influencia en la producción de pastos; miembros que trabajan en la unidad agrícola $54 \%$ son padres de familia y $16 \%$ son madres de familia responsables de cosecha; con respecto a la disponibilidad de agua para riego el $56 \%$ no disponen. En el aspecto de la producción para mejora de calidad de vida se evaluó la variable del rendimiento de pastos mejorados $28 \%$ tiene un rendimiento 30 - 35 tonelada por hectárea con seis cosechas al año y la mejora de ingresos económicos de los productores, el $40 \%$ es regular y $53 \%$ es bueno; en la contratación de hipótesis no existe diferencia estadística significativa. Se concluye, la producción de pastos no influye favorable en el impacto económico significativamente en el productor.

Palabras clave: Impacto económico; Pastos mejorados; Perenne

ABSTRACT

RESUMO

The research objective was to evaluate the economic impact of improved pasture production on agricultural producers, the methods used were deductive - inductive with its documentary review technique and their respective instruments of descriptive statistics, the results of the social aspects; in the variable level of education of producers has little influence on the production of pasture; members working in the agricultural unit $54 \%$ are fathers and $16 \%$ are mothers responsible for harvest; with respect to the availability of water for irrigation $56 \%$ do not have it. In the aspect of production to improve the quality of life, the variable of improved pasture yield was evaluated; $28 \%$ have a yield of 30-35 tons per hectare with six harvests per year and the improvement of economic income of the producers, $40 \%$ is regular and $53 \%$ is good; in the contracting of hypotheses there is no significant statistical difference. It is concluded that pasture production does not have a favorable influence on the economic impact significantly on the producer.

Key words: Economic impact; Improved pastures; Perennial
O objetivo da pesquisa foi avaliar o impacto econômico da produção de pastagens melhoradas nos produtores agrícolas, os métodos utilizados foram dedutivos indutivos com sua técnica de revisão documental e seus respectivos instrumentos de estatística descritiva, os resultados dos aspectos sociais; no nível de educação variável dos produtores tem pouca influência na produção de pastagens; os membros que trabalham na unidade agrícola 54\% são pais de família e $16 \%$ são mães de família responsáveis pela colheita; com relação à disponibilidade de água para irrigação 56\% não a têm. No aspecto da produção para melhorar a qualidade de vida, foi avaliada a variável de melhor rendimento de pastagem; $28 \%$ têm um rendimento de 30-35 toneladas por hectare com seis colheitas por ano e a melhoria da renda econômica dos produtores, $40 \%$ é regular e $53 \%$ é boa; no teste de hipóteses não há diferença estatística significativa. Conclui-se que a produção de pastagens não influencia favoravelmente o impacto econômico significativo sobre o agricultor.

Palavras-chave: Impacto econômico; Pastagens melhoradas; Perene 


\section{INTRODUCCIÓN}

Las campañas de instalación de pastos mejorados contribuyen significativamente a la mejora de calidad de crianzas de animales menores y mayores, como consecuencia el aumento significa mayores ingresos económicos en las familias productores de pastos y por ende la mejora de su calidad de vida, de la misma manera manifiesta López-Carrasco (1) La producción de pastos mejorados aumenta producción de leche por consecuencia que significa mejores ganancias económicas y una mejor consideración social, según los estudios de Ríos (2). En los distritos de la provincia de Yarowilca, se desarrollaron proyectos productivos de instalación y manejo de pastos cultivados, financiados por el gobierno regional de Huánuco y al finalizar la inversión dejaron más 600 hectáreas de pastos mejoradas en diferentes especies según Ponce (3). Por consecuencia las familias mejoran sus ingresos económicos, la investigación proporciona la evaluación de impacto económico en las familias agropecuarios con de la siembra y producción de pastos mejorados. La investigación tuvo como objeto evaluar el impacto económico con la producción de pastos mejorados perennes en los productores agropecuarios; con la hipótesis formulada la producción de pastos mejorados perennes influye favorablemente en el impacto económico de los productores agropecuarios. La presente investigación es importante porque ha permitido conocer la mejora de calidad de vida con la producción de pastos mejorados y así proponer correctivos para la toma de decisiones que repercutirán en beneficio los productores agropecuarios.

\section{MÉTODO}

Para el desarrollo del estudio se usaron el método inductivo y deductivo con su técnica de análisis de la información de la revisión documental y sus respectivos instrumentos, el análisis-síntesis, la técnica la matemática y sus instrumentos de la estadística descriptiva.

La investigación se ubicó en el diseño no experimental porque no se manipularon las variables; transeccional porque fueron recolectado los datos en un solo momento; descriptivo porque fue descrita la realidad de la mejora de calidad de vida de las familias agropecuarios. La población fueron 1500 productores, el tipo de muestra fue el aleatorio estratificado en tres distritos de Chavinillo (17), Obas (40) y Aparicio Pomares (43) con cien (100) productores agropecuarios.

Para probar la hipótesis se construyó un cuestionario, teniendo en cuenta los indicadores y dimensiones de la variable independiente, impacto económico en los productores agropecuarios de los distritos de la provincia. El procesamiento de datos obtenidos fue a través del instrumento de distribución de frecuencias, las hipótesis se realizaron con la prueba no paramétrica ChiCuadrado que determinó la relación entre variables de estudio.

\section{RESULTADOS Y DISCUSIÓN}

La obtención de los resultados del trabajo de investigación fueron aplicado los instrumentos de recolección de la información, se procedió a realizar los tratamientos correspondientes para el análisis de los mismos, por cuanto la información que se consiguió es la que se 
indica en la presentación de los resultados a las cuales llevó este estudio.

Aspectos sociales para la producción de pastos mejorados

En la Tabla 1 se muestra que el mayor porcentaje de productores agropecuarios tienen primaria completa, el cual está representado por $31 \%$ el mismo que lo constituyen 30 productores con secundaria completa, existe productores que tiene superior completa y está representado por $21 \%$ y productores que no tienen instrucción. En esta variable se afirma que los productores de pastos mejorados están conformados por personas que tienen diferentes niveles de instrucción.

Tabla 1. Nivel de Instrucción de los productores de los distritos de Chavinillo, Obas y Aparicio pomares se detalla a continuación.

\begin{tabular}{lccc}
\hline \multicolumn{1}{c}{ Nivel de Instrucción } & Frecuencia & $\mathbf{f} \%$ & $\mathbf{f}(\%) \uparrow$ \\
\hline Superior Completa & 21 & 21 & 21 \\
Secundaria Completa & 30 & 30 & 51 \\
Primaria Completa & 31 & 31 & 82 \\
Sin Instrucción & 18 & 18 & 100 \\
TOTAL & 100 & 100 & \\
\hline
\end{tabular}

\section{Miembros que trabajan en la unidad agrícola}

En la Tabla 2 se muestra que el mayor porcentaje de productores agropecuarios que trabajan en la unidad agrícola son padres de familia, y está representado por $54 \%$ el mismo que lo siguen son hijos y madres de familia. En esta variable se afirma que el $50 \%$ son los responsables para la producción de pastos mejorados los padres de familia y los hijos, madres, otros también participan en la producción de pastos con las diferentes actividades agronómicas.

Tabla 2. Los resultados de los miembros que trabajan en la unidad agrícola se muestran a continuación.

\begin{tabular}{lccc}
\hline $\begin{array}{l}\text { Miembros que trabajan en } \\
\text { la unidad agrícola }\end{array}$ & Frecuencia & $\mathbf{f} \%$ & $\mathbf{f ( \% ) \uparrow}$ \\
\hline Padres de familia & 54 & 54 & 54 \\
Madres de familia. & 16 & 16 & 70 \\
Hijos. & 21 & 21 & 91 \\
Otros. & 9 & 9 & 100 \\
TOTAL & 100 & 100 & \\
\hline
\end{tabular}


Aspectos de producción para mejora de calidad de vida del productor

En la Tabla 3 se observa que el mayor porcentaje de productores de pastos mejorados, y está representado por un $28 \%$ que tienen un rendimiento de pastos de 30 a 35 ton/ha de alfalfa, el mismo que $24 \%$ de productores tienen pastos asociados con 30 a 35 ton/ha. En esta variable se afirma que la producción de pastos mejorados asociado y no asociado tiene un rendimiento más de 30 a $50 \%$, en condiciones climáticas frías y los productores realizan las labores agronómicas y culturales adecuadas.

Tabla 3. El rendimiento de los pastos mejorados los resultados de los rendimientos por hectárea de pastos mejorados que producen los producen se detallan a continuación.

\begin{tabular}{lccc}
\hline \multicolumn{1}{c}{ Rendimiento en Ton/ha. } & Frecuencia & $\mathbf{f} \%$ & $\mathbf{f ( \% )}$ \\
\hline Alfalfa 25-30 Ton/Ha. & 14 & 14 & 14 \\
Alfalfa 30 - 35 Ton/Ha. & 28 & 28 & 42 \\
Alfalfa 35 -40 Ton/Ha. & 15 & 15 & 57 \\
Alfalfa + Dactylis 25 - 30 Ton/Ha. & 12 & 12 & 69 \\
Alfalfa + Dactylis 30 - 35 Ton/Ha. & 24 & 24 & 93 \\
Alfalfa + Dactylis 35 - 40 Ton/Ha. & 5 & 5 & 98 \\
Más de 40 Ton/Ha. & 2 & 2 & 100 \\
TOTAL & 100 & 100 & \\
\hline
\end{tabular}

\section{Mejora de ingresos económicos}

Se muestra que el mayor porcentaje de productores agropecuarios que mejoraron sus ingresos económicos con un calificativo de bueno, el cual está representado por 35\% y el mismo que respondieron de la mejora sus ingresos económicos con el calificativo de regular, En esta variable se afirma que los productores de pastos mejorados han mejorado sus condiciones de vida en servicios básicos, educción de sus hijos y por consecuencia el impacto económico es favorable en los productores de los distritos de la provincia de Yarowilca.

Tabla 4. Mejora de ingresos económicos Los resultados de la mejora de los ingresos económicos en los productores agropecuarios se detallan a continuación.

\begin{tabular}{lccc}
\hline $\begin{array}{c}\text { Mejora de ingresos } \\
\text { económicos }\end{array}$ & Frecuencia & $\mathbf{f} \%$ & $\mathbf{f ( \% )}$ \\
\hline Muy malo & 1 & 1 & 1 \\
Malo & 2 & 2 & 3 \\
Regular & 40 & 40 & 43 \\
Bueno & 53 & 53 & 96 \\
Muy bueno & 4 & 4 & 100 \\
TOTAL & 100 & 100 & \\
\hline
\end{tabular}




\section{Discusión}

Los resultados de aspectos de la producción en la mejora de la calidad de vida del productor el rendimiento de los pastos mejorados de la especie de alfalfa, $28 \%$ de productores sus pastos tienen rendimientos de 30 a 35 ton/ha/corte en materia verde, habiendo ligera diferencia entre la producción de otro productores que tienen rendimientos superiores o inferiores, la producción de pastos en la provincia de Yarowilca no es muy adecuado, por diferentes factores como: en clima es frío, en suelos pendientes, la variedad de pasto sembrado, el agua para riego, el abonamiento. Estos rendimientos son inferiores al trabajo de investigación de Álvarez (4), donde evaluó variables que determinan el crecimiento y rendimiento de diez variedades de alfalfa a intervalos de corte definidos estacionalmente, encontrando el mayor rendimiento variedad Júpiter (14.510 $\mathrm{kg}$ de materia seca por hectarea) y el menor rendimiento fue para la variedad San Miguelito (7.890 kg de materia seca por hectárea). En condiciones y realidades muy diferentes con respecto a la provincia de Yarowilca, de la misma manera se compara con Flores et al. (5), donde probó determinación de la producción de forraje de alfalfa y propiedades químicas del suelo como respuesta a la aplicación de fertilizante orgánico.

En la variable de la mejora de los ingresos económicos con la producción de pastos mejorados no es significativo estadísticamente, estos datos son muy diferentes al comparar por diario en El mercurio.pe (6), donde publico los resultados del proyectos "mi chacra emprendedora" con 517 familias beneficiarias, en la comunidad de Conga donde brindó asistencia técnica personalizado, en siembra de pastos mejorados desde la instalación, manejo y conservación, así mismo se compara con
Ríos (2), donde investigó un caso económico, de un proyecto exitoso de capacitación y asistencia técnica en Cajamarca, evaluado el efecto económico positivo en las familias de los productores sus logros principales fueron incremento de la producción por vaca en 25 por ciento, basado en el alto rendimiento de los pastos.

\section{CONCLUSIONES}

Se concluye en relación al objetivo propuesto al inicio que la producción de pastos mejorados estadísticamente, la influencia no es muy significativo en la mejora de ingresos económicos y como consecuencia el impacto económico es poco favorable en los productores agropecuarios de los distritos de la provincia de Yarowilca.

Además, el nivel de instrucción en los agricultores de la provincia de Yarowilca tiene poca influencia en el manejo y rendimiento de pastos mejorados.

Por lo que, la disponibilidad de terreno aptos para la producción de pastos y la disponibilidad del agua para riego son escasas para los productores agropecuarios de la provincia de Yarowilca.

En el entendido, que las especies de pastos mejorados en parcelas de los productores de la provincia de Yarowilca es su mayoría es alfalfa con promedios de 6 a 7 cosecha por año.

Destacando, en la provincia de Yarowilca los rendimientos de los pastos mejorados oscilan entre 30 a 35 toneladas por hectárea en promedio.

Obteniendo que la producción de pastos mejorados ha influido favorablemente en el nivel de educación de sus hijos, condiciones de vivienda, servicios básicos, y el nivel de ingresos económicos de los productores de la provincia de Yarowilca. 


\section{REFERENCIAS BIBLIOGRÁFICAS}

1 López-Carrasco C, Rodríguez R, Robledo JC.Efecto de la fertilización fosfórica en la transformación a pastizal de un cultivo forrajero en la Campana de Oropesa (Toledo). Actas de la XXXIX Reunión Científica de la SEEP, 1999; pp.407-412.

2. Allen R, Pereira L. Guías para la determinación de los requerimientos de agua de los cultivos Roma. 2006; 110p.

3. Ponce Gonzáles. Reunión sobre ajuste tecnológico pecuario para los pequeños productores - Colombia (1984) 6-9;40-57 p.

4. Álvarez P. Evaluación cuantitativa de diez variedades de alfalfa (Medicago sativa L.), México. 2013; 79. p
5. Flores J, Reyes R, Vergara J, Flores I, Bahena M. Efecto de fertilizante orgánico, inorgánico y su combinación en la producción de alfalfa y propiedades químicas del suelo-México. 2013; 213p

6. ELMERCURIO.PE. Agradecen a FONCODES por su apoyo con programa Haku Wiñay en línea, consultado el 17 de febrero. 2015; Disponible en: http://www.elmercurio.pe/ index.php/regionales/14696-agradecen-afoncodes-por-su-apoyo-con-programa-hakuwinay 